\title{
Cytoskeleton/Tumor Biology
}

National Cancer Institute

\section{Source}

National Cancer Institute. Cytoskeleton/T umor Biology. NCI Thesaurus. Code C19625.

The study of the role of the cytoskeleton in the development and treatment of tumors and cancer. 